\title{
Bullying y Autoestima en los Estudiantes de la Universidad Nacional José Faustino Sánchez Carrión, Huacho
}

\author{
Bullying and Self-Esteem in Students of the University Jose Faustino Sanchez Carrion, Huacho
}

Maritza Isabel Martínez Loli ${ }^{1}$, Norma Adela Moreno Rivera', Perfecta Zobeida Fernández Guzmán², María Rosario Meza Aguirre $^{3}$, Marcelo Gumercindo Zúñiga Rojas', Bethsabeth Melosich Aviles', Luz Mery Tadeo Sifuentes', Jaqueline Andrea Velásquez Fernández ${ }^{2}$, Marina Olinda Adauto Retuerto ${ }^{2}$, Fernando Francisco, Guillermo Agama ${ }^{1}$

\section{RESUMEN}

Objetivo: Identificar la relación entre bullying y la autoestima en los estudiantes de la Universidad Nacional José Faustino Sánchez Carrión. Métodos: Fue una investigación básica de nivel correlacional, corte transversal. La población estuvo formada por 993 estudiantes de 10 escuelas Profesionales de la Universidad. La muestra de tipo no probabilístico ascendió a 232 estudiantes que equivale a 23,33\% del total. Para la recolección de datos se empleó el Cuestionario sobre bullying universitario que comprende tres dimensiones: rol del agresor, rol de víctima y rol del observador y consta de 20 ítems; elaborado por el equipo de investigadores validando el instrumento a través de juicio de expertos obteniendo 0,950 y para la confiabilidad la aplicación de una prueba piloto arrojando el alfa de cronbach de 0,825; y la Escala de Autoestima de Rosemberg que consta de 10 ítems, comprende tres dimensiones: Pensamientos, sentimientos y conductas, validado por el autor y arrojando un alfa de cronbach de 0,757. La prueba de hipótesis se utilizó la prueba de Friedman (Chi cuadrado de Pearson) Para el procesamiento de la información se utilizó el programa estadístico SPSS versión 20. Resultados: El 57,3\% manifestaron la existencia de prácticas de Bullying en los estudiantes universitarios, el 53\% presentan autoestima media, de los cuales el $29,3 \%$ señalan practicas Bullying y tienen autoestima media. Se encontró que el $93,5 \%$ son jóvenes de los que el $68,7 \%$ asumen el rol de agresores y por último, los universitarios varones en un $59,3 \%$ manifiestan ausencia de Bullying y presentan autoestima media a diferencia de las estudiantes mujeres que el $64,2 \%$ señalaron que existe Bullying en la universidad, el 51,4\% presentan autoestima media de los cuales el 32,4\% perciben el Bullying y tienen autoestima media Conclusión: Existe relación entre el Bullying y la Autoestima en los estudiantes de la Universidad Nacional José Faustino Sánchez Carrón.

Palabras clave: Bullying, autoestima, agresor, víctima, observador, estudiantes universitarios.

\section{ABSTRACT}

Objective: To identify the relationship between bullying and self-esteem in students at José Faustino Sanchez Carrion National University. Methods: It was a basic research of correlational level, cross-section. The population consisted of 993 students from 10 Professional Schools of the University. The non-probabilistic sample amounted to 232 students equivalent to $23.33 \%$ of the total. For data collection was used the Questionnaire about University Bullying which includes three dimensions: role of the aggressor, role of the victim and role of the observer and it consists of 20 items; developed by the research team validating the instrument through expert judgment obtaining 0.950 and for the reliability the application of a pilot test throwing a Cronbach Alpha of 0,825 and the with a confidence level of 0, 809 and Rosenberg Self-Esteem Scale that consists of 10 items, includes three dimensions: thoughts, feelings and behaviors; validated by the author and throwing a Cronbach Alpha of 0.757. For hypothesis test was used Friedman test (Pearson Chi square), for the information processing was used the statistical program SPSS version 20. Results: $57.3 \%$ reported the existence of bullying practices in university students, $53 \%$ have average self-esteem, of which $29.3 \%$ indicates Bullying practices and have average selfesteem. Also, $93.5 \%$ are young of which $68.7 \%$ assume the role of aggressors and finally, $59.3 \%$ of male students show absence of bullying and average self-esteem to difference of female students who $64,2 \%$ indicated that bullying exists in the university, $51.4 \%$ have average self-esteem of which $32.4 \%$ perceive bullying and have average self-esteem Conclusion: There is relationship between bullying and self-esteem in students at José Faustino Sanchez Carrion National University.

Keywords: bullying, self-esteem, aggressor, victim, observer, university students.

Facultad de Ciencias Sociales. Universidad Nacional José Faustino Sánchez Carrión. Huacho, Perú.

${ }^{2}$ Facultad de Educación. Universidad Nacional José Faustino Sánchez Carrión. Huacho, Perú.

${ }^{3}$ Facultad de Derecho y Ciencias Políticas. Universidad Nacional José Faustino Sánchez Carrión. Huacho, Perú. 


\section{INTRODUCCIÓN}

El bullying se ha convertido en una problemática social - educativa que afecta no solo a estudiantes de los niveles de educación primaria y educación secundaria, sino también está afectando a los universitarios a través de las diversas formas de violencia que se manifiestan en conductas reiterativas convirtiéndose en acoso u hostigamiento lo que conlleva a que el estudiante acosado u hostilizado desarrolle miedos de ingresar y / o permanecer en los ambientes de la universidad, así mismo al percibir un sentimiento de rechazo o de burla va menoscabando su autoimagen y su auto concepto y genera irregularidad e inestabilidad emocional consecuentemente desarrolla una baja autoestima y posteriormente influirá en su aprendizaje y bajo rendimiento. Entendemos por Bullying como una forma de maltrato, normalmente intencionado, perjudicial y persistente de un estudiante o grupo de estudiantes, hacia otro compañero, generalmente más débil, al que convierte en su víctima habitual, sin que medie provocación y, lo que quizá le imprime el carácter más dramático, la incapacidad de la víctima para salir de esa situación, acrecentando la sensación de indefensión y aislamiento (Cerezo, 2009). Por otro lado, existen muchas teorías que tratan de explicar el bullying; en esta investigación se considera la Teoría del Aprendizaje Social de Bandura (1973) enmarcado dentro del modelo cognitivoconductual que señala que la agresión tiene muchos determinantes y diversos propósitos (Bandura, 1973) y por ello la Teoría del Aprendizaje Social pretende ofrecer un modelo explicativo más amplio que el de "FrustraciónAgresión", para abarcar las condiciones que regulan todas las facetas de la agresión, sea individual o colectiva. Esta teoría define a la agresión como la conducta que produce daños a la persona y la destrucción de la propiedad (Bandura y Ribes, 1975); y ese daño puede adoptar formas psicológicas (devaluación y degradación) o forma física.

Bandura (1973) al referirse específicamente acerca del origen de la agresión, menciona al aprendizaje por observación donde existen muchas influencias (familiares, sub culturales y modelamiento simbólico), a la ejecución reforzada o aprendizaje por experiencia directa y por último a los determinantes estructurales.

Concluye la teoría, que las influencias familiares son las que mayor repercusión tienen en la vida de las personas, por su disponibilidad de modelos y por las carencias que pueden ocasionar; muchas investigaciones avalan este punto como Olweus (1993), Loeber y Dishion, (1983), Patterson y Stouthamer-Loeber, (1984) y Patterson, (1986). Se destaca el modelamiento simbólico a través de la televisión, ya que los patrones de respuesta trasmitidos gráfica o verbalmente, pueden aprenderse a través de la observación de una manera tan eficaz como aquellos presentados mediante demostraciones sociales (Moreno (1999). Bandura, Ross y Ross, 1963 a; Bandura y Mischel, 1965).

Es preocupante observar o escuchar testimonios de jóvenes universitarios que son víctima del bullying, e incluso se conoce que en el año 2012, hubo 15 intentos de suicidio que estaba asociado al bullying en una universidad del norte del país, lo que nos lleva a inferir que este sector está siendo desatendido por sus docentes, padres de familia y las autoridades, es por estas razones que nuestro equipo de investigación ha decidido estudiar bajo el rigor científico este tema para poder detectar si hay bullying o no, y con los resultados a que se arribe realizar propuestas de intervención para promover programas de prevención evitando la práctica de conductas agresivas, tanto físicas como verbales.

En tal sentido se ha revisado investigaciones a nivel internacional y nacional, tales como: García, Moncada y Quinteros (2013). Estudiaron sobre El Bullying y el suicidio en el escenario universitario. Estudio realizado en Colombia, que tuvo como objetivo presentar los resultados obtenidos en el rastreo bibliográfico y documental de las principales contribuciones de la literatura científica sobre el tema. Es así que se abre un nuevo panorama en el conocimiento del fenómeno de intimidación y el ciber-acoso en escenarios universitarios. Intimidación, asedio, hostigamiento son términos que refieren el acosos escolar o bullying, entendido como un fenómeno polimorfo, multicausal, complejo, que se presenta además, como una de las causas de suicidio en la población juvenil. Por ello los autores manifiestan que es importante reconocer el origen del término bullying, y contextualizarlo en el ámbito universitario con todas las implicancias que conlleva desde sus causas, consecuencias, relación con el suicidio, rol de la familia, prevención e intervención. 
Benítez, Muñoz, Fernández y Berbén (2005), realizaron una investigación sobre Conocimiento y actitud del maltrato entre alumnos (Bullying) de los futuros docentes de educación infantil, primaria y secundaria, cuyo objetivo fue analizar los conocimientos y actitudes que poseen los futuros docentes de Educación Infantil, Primaria y Secundaria sobre la violencia entre escolares. Se centra la atención en el análisis de las estrategias de afrontamiento del problema preferidas por el futuro profesorado para resolver el problema del maltrato entre iguales. Finalmente, analizan las demandas de formación que sobre el fenómeno estudiado realizan los futuros docentes. La muestra está formada por 373 estudiantes de las Diplomaturas de Educación Infantil y Primaria y alumnos del Curso para la obtención del Certificado de Aptitud Pedagógica. Los resultados obtenidos indicaron que los participantes conocen el problema y sus efectos aunque se hace necesaria mayor información y formación específica para afrontar el fenómeno. Demandaron la formación de equipos para trabajar con víctimas, agresores, familias y observadores. Del mismo modo, quedo latente la necesidad de trabajar para prevenir el problema y no centrarse solamente en paliar sus efectos.

Romero, Cruz, Gallardo y Peñacoba, (2013). Desarrollaron un estudio sobre Cómo promocionar la salud y el bienestar en la comunidad universitaria. Universidad Rey Juan Carlos. Las Universidades deben contribuir a promocionar y potenciar los valores que permiten el bienestar, la autonomía y el completo desarrollo de las personas que en ella estudian y trabajan. En este trabajo se describió una de las iniciativas en las que participaron 181 usuarios que voluntariamente accedieron a una modalidad de intervención individual ó grupal de ocho sesiones en función de sus necesidades auto detectadas. Los resultados mostraron evoluciones significativas pre y post en las cuatro variables evaluadas: autoeficacia, autoestima, afecto positivo y negativo, manteniéndose las mejoras en el seguimiento, sin diferencias en cuanto a colectivo y sexo, pero sí con respecto a la modalidad terapéutica (individual vs. terapéutica) con respecto al afecto. Se señalaron las implicaciones prácticas de esta iniciativa como un medio de promocionar la salud y el bienestar en el contexto universitario.

Gómez-Talavera, (2013) Estudió sobre Nivel de agresión entre pares de estudiantes de una Universidad de Lima Metropolitana. Es un estudio que tuvo por objetivo: Conocer el nivel de agresión en universitarios, empleando la Escala de Agresión entre Pares. Material y Métodos: Trabajo con una muestra de 235 universitarios de Lima, Perú; de 16 a 19 años. Se utilizó la Escala de Agresión que está compuesta por cuatro sub-escalas. A todos se les explicó y pidió que voluntariamente completasen los cuestionarios. El material fue manejado exclusivamente por el equipo de investigadores. Resultados: Se encontró que las influencias externas de la violencia alcanzaron un $10,95 \%$, el $9,42 \%$, mostró una actitud conciliadora ante un hecho de agresión. Y llego a la siguiente conclusión: Se halló una tendencia a armonizar ante situaciones de agresión. Propuso realizar otro estudio en el que se correlacione los resultados con el nivel vocacional del estudiante.

Con respecto a la Autoestima, Bonet (1997) nos dice que la autoestima es un conjunto de actitudes que dependen de las percepciones, pensamientos, evaluaciones, sentimientos y tendencias de comportamiento dirigidas hacia nosotros mismos, hacia nuestra manera de ser y de comportarnos, y hacia los rasgos de nuestro cuerpo y nuestro carácter.

Por último, Tarazona (2005) obtuvo resultados que muestran respecto a la autoestima, que las mujeres de escuela pública tuvieron el menor nivel, los varones de colegio privado, el mayor nivel; finalmente los varones de escuela pública y las mujeres de escuela privada tuvieron un similar nivel intermedio.

\section{MATERIALES Y METODOS}

Es una investigación descriptiva correlacional; cuyo objetivo es recolectar información relevante en una muestra respecto a un mismo fenómeno, variables o aspecto de interés y luego caracterizar este fenómeno en base a la relación de dos o más variables (Sánchez, 2006). La población estuvo formada por 993 estudiantes del Octavo ciclo de las 12 facultades de la universidad y la muestra fue de 232 estudiantes que equivale al $23,33 \%$ de las Escuelas profesionales de Bromatología y Nutrición, Ciencias de la comunicación, Derecho y Ciencias Políticas, Economía y Finanzas, Educación Inicial, Educación Primaria, Enfermería, Industrias Alimentarias, Sociología y Trabajo Social. 
Para la obtención de datos referentes a la unidad de análisis, se utilizó el Cuestionario sobre Bullying Universitario que comprende tres dimensiones: rol del agresor, rol de víctima y rol del observador y consta de 20 ítems; elaborado por el equipo de investigadores validado a través de un juicio de expertos y una prueba piloto con un nivel de confiabilidad de 0 , 809.

Tabla 1. Categorización de autoestima

\begin{tabular}{lc}
\hline Dimensiones & Categorización \\
\hline Autoestima Elevada & 10 a 28 \\
Autoestima Media & 29 a 36 \\
Autoestima Alta & 37 a 40 \\
\hline
\end{tabular}

Fuente: Elaboración propia según cuestionario administrado a los alumnos de la U.N.J.F.S.C. 2015

\section{RESULTADOS}

Para conocer la fiabilidad de ambos instrumentos: Cuestionario de Bullying Universitario y Escala de Autoestima de Rosemberg, se aplicó una prueba piloto; arrojando el Alfa de Cronbach de 0,825 y 0,757 respectivamente que significa una aceptable confiabilidad.

Tabla 2. Prueba de Alfa de Cronbach de Bullying y Autoestima

\begin{tabular}{cccc}
\hline & Bullying & & Autoestima \\
\hline $\mathrm{N}$ de & Alfa de & $\mathrm{N}$ de & Alfa de \\
elementos & Cronbach & elementos & Cronbach \\
20 & 0,825 & 10 & 0,757
\end{tabular}

Con respecto a la edad de los estudiantes, Tabla 3 se encontró que la edad promedio es de 21,7 la edad estas oscilan entre 18 a 35 años y la variación de las edades es de 5.1. Por otro lado de acuerdo al ciclo de vida tenemos que el $93,5 \%$ son jóvenes entre 20 y 30 años de edad, el $4,7 \%$ están en la adolescencia tardía entre 18 y 19 años y solo el $1,7 \%$ son adultos de 30 a 35 años de edad.

Tabla 3. Grupo de edades según el ciclo de vida

\begin{tabular}{ccc} 
& $\mathbf{n}$ & $\%$ \\
\hline Adolescentes & 11 & 4,7 \\
Jóvenes & 217 & 93,5 \\
Adultos & 4 & 1,7 \\
Total & 232 & 100,0
\end{tabular}

Con respecto al género tenemos que el $74,6 \%$ son de sexo femenino y solo $25,4 \%$ son de sexo masculino, demostrando la predominancia de mujeres.

En relación a las escuelas profesionales tenemos que la mayoría de estudiantes el $20,3 \%$ pertenecen a la escuela Profesional de Economía y Finanzas y existe menor porcentaje de 3,9\% respectivamente de las escuelas profesionales de Ingeniería Alimentaria y Educación Primaria. (Figura 1).

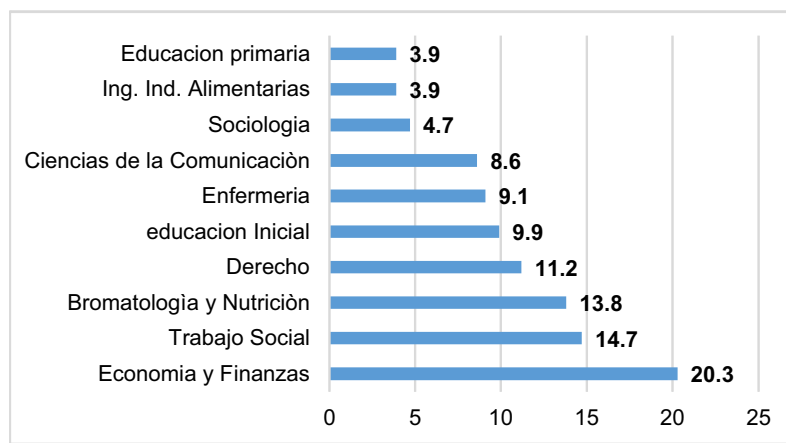

Figura 1. Distribución de la población según Escuelas Profesionales. UNJFSC.2015

En la Tabla 4, se comparan las frecuencias y porcentajes obtenidos de acuerdo a roles de los estudiantes en el bullying; asimismo que el $72,8 \%$ manifiestan haber agredido alguna vez a sus compañeros, el $75,4 \%$ señalan haber recibido Bullying de parte de sus compañeros y $68,5 \%$ menciona que nunca interviene en un agresión donde él es solo observador.

Tabla 4. Distribución de frecuencia y porcentajes de roles en el Bullying

\begin{tabular}{ccccccc}
\hline \multirow{2}{*}{ Respuestas } & \multicolumn{2}{c}{ Agresor } & \multicolumn{2}{c}{ Victima } & \multicolumn{2}{c}{ Observador } \\
\cline { 2 - 7 } & $\mathbf{N}$ & $\%$ & $\mathbf{N}$ & $\%$ & $\mathbf{N}$ & $\%$ \\
\hline $\begin{array}{c}\text { Si (agrede, recibe, } \\
\text { interviene) }\end{array}$ & 169 & 78,8 & 175 & 75,4 & 73 & 31,5 \\
$\begin{array}{c}\text { No (agrede, recibe, } \\
\text { interviene) }\end{array}$ & 63 & 27,2 & 57 & 24,6 & 159 & 68,5 \\
$\quad$\begin{tabular}{c} 
Total \\
\hline
\end{tabular} & 232 & 100 & 232 & 100 & 232 & 100 \\
\hline
\end{tabular}

Asimismo se tiene datos agrupados donde el $57,3 \%$ señala que existen prácticas permanentes de Bullying entre los estudiantes de sus respectivas escuelas profesionales y el $42,7 \%$ manifiestan que las prácticas de Bullying están ausentes en los estudiantes universitarios. Por otro lado, se encuentra diferencias en los niveles de autoestima, es así que el $53 \%$ presenta autoestima media, el $25,4 \%$ autoestima baja y el $21,6 \%$ autoestima alta. Tabla 5. 
Tabla 5. Distribución de frecuencias y porcentajes según niveles de Autoestima

\begin{tabular}{lrr}
\hline Niveles & n & $\%$ \\
\hline Autoestima Baja & 59 & 25,4 \\
Autoestima Media & 123 & 53,0 \\
Autoestima Alta & 50 & 21,6 \\
Total & 232 & 100,0 \\
\hline
\end{tabular}

\section{CONTRASTACION DE LAS HIPOTESIS}

\section{Hipótesis General}

La Tabla 6 , nos señala que el $57,3 \%$ manifestaron la presencia de prácticas de Bullying en la universidad, así como; el 53,0\% presentan autoestima media y un $29,3 \%$ manifiestan la existencia de prácticas de bullying y tienen autoestima media.

Tabla 6. Distribución de frecuencias y porcentajes de Bullying y nivel de autoestima

\begin{tabular}{|c|c|c|c|c|c|c|c|c|}
\hline \multirow{3}{*}{ Bullying } & \multicolumn{6}{|c|}{ Nivel de autoestima } & \multicolumn{2}{|c|}{ Total } \\
\hline & \multicolumn{2}{|c|}{ Baja } & \multicolumn{2}{|c|}{ Media } & \multicolumn{2}{|c|}{ Alta } & \multirow{2}{*}{ n } & \multirow{2}{*}{$\%$} \\
\hline & $\mathbf{n}$ & $\%$ & $n$ & $\%$ & $n$ & $\%$ & & \\
\hline & 33 & 14,2 & 68 & 29,3 & 32 & 13,8 & 133 & 57,3 \\
\hline Ausencia de Bullying & 26 & 11,2 & 55 & 23,7 & 18 & 7,8 & 99 & 42,7 \\
\hline Total & 59 & 25,4 & 123 & 53,0 & 50 & 21,6 & 232 & 100 \\
\hline
\end{tabular}

Se docimó las hipótesis general aplicando la Prueba Friedman (Chi cuadrado de Pearson) obteniendo 58,778 , con un p-valor de 0,000 ; lo cual indica que existen evidencias estadísticas, para señalar la relación altamente significativa entre el Bullying y la Autoestima en los estudiantes de la Universidad Nacional José Faustino Sánchez Carrión.

\section{Hipótesis especifica 1}

Se observa en la Tabla 7, que los estudiantes universitarios de acuerdo al rol de agresor se encuentran distribuidos de la siguiente manera: El 3,9\% son adolescentes, $68,7 \%$ son jóvenes y no existe ningún adulto que ha manifestado la agresión a sus compañeros.
Tabla 7. Grupo de edades de acuerdo al ciclo de vida según el rol del agresor

\begin{tabular}{|c|c|c|c|c|c|c|}
\hline \multirow{3}{*}{$\begin{array}{l}\text { Grupo de Edades } \\
\text { Según Ciclo Vital }\end{array}$} & \multicolumn{4}{|c|}{ Rol del Agresor } & \multicolumn{2}{|c|}{ Total } \\
\hline & \multicolumn{2}{|c|}{$\begin{array}{l}\text { Nunca } \\
\text { agrede }\end{array}$} & \multicolumn{2}{|c|}{$\begin{array}{l}\text { Agrede a sus } \\
\text { compañeros }\end{array}$} & $\mathbf{n}$ & $\%$ \\
\hline & $\mathbf{n}$ & $\%$ & $\mathbf{n}$ & $\%$ & & \\
\hline Adolescentes & 2 & 0,9 & 9 & 3,9 & 11 & 4,8 \\
\hline Jóvenes & 57 & 24,8 & 158 & 68,7 & 215 & 93,5 \\
\hline Adultos & 4 & 1,7 & 0 & 0,0 & 4 & 1,7 \\
\hline Total & 63 & 27,4 & 167 & 72,6 & 230 & 100 \\
\hline
\end{tabular}

Se docimó las hipótesis especifica 1 aplicando la Prueba de Friedman (Chi cuadrado de Pearson), obteniendo 38,629, con un p-valor de 0,000 ; lo cual indica que existen evidencias estadísticas, para señalar la relación altamente significativa entre el Bullying y la Autoestima en las diferentes etapas del ciclo de vida de los estudiantes de la Universidad Nacional José Faustino Sánchez Carrión; presentándose una mayor práctica en los jóvenes universitarios.

\section{Hipótesis especifica 2}

En la Tabla 8, se observa que el 59,3\% manifestaron ausencia de Bullying y presentan un nivel de autoestima media en los estudiantes varones y el $40,7 \%$ señalan que si existe prácticas de Bullying. Por último, el $32,2 \%$ manifiestan la ausencia de Bullying y tienen autoestima media.

Tabla 8. Distribución de frecuencias y porcentajes de Bullying y autoestima en varones

\begin{tabular}{|c|c|c|c|c|c|c|c|c|}
\hline \multirow{3}{*}{$\begin{array}{l}\text { Practicas de } \\
\text { Bullying en } \\
\text { Varones }\end{array}$} & \multicolumn{6}{|c|}{$\begin{array}{c}\text { Nivel de autoestima en } \\
\text { varones }\end{array}$} & \multicolumn{2}{|c|}{ Total } \\
\hline & \multicolumn{2}{|c|}{ Baja } & \multicolumn{2}{|c|}{ Media } & \multicolumn{2}{|c|}{ Alta } & \multirow{2}{*}{$\mathbf{n}$} & \multirow{2}{*}{$\%$} \\
\hline & $n$ & $\%$ & $n$ & $\%$ & $n$ & $\%$ & & \\
\hline Prácticas de Bullying & 2 & 3,4 & 16 & 27,1 & 6 & 10,2 & 24 & 40,7 \\
\hline Ausencia de Bullying & 9 & 15,3 & 19 & 32,2 & 7 & 11,9 & 35 & 59,3 \\
\hline Total & 11 & 18,6 & 35 & 59,3 & 13 & 22 & 59 & 100 \\
\hline
\end{tabular}

En la Tabla 9, se observa que el $64,2 \%$ manifiestan existencia de prácticas de Bullying, solo el 35,8\% afirman la existencia de dichas prácticas, así como el $51,4 \%$ presentan autoestima media. Por último el $32,4 \%$ señalaron prácticas de Bullying y la vez tiene autoestima media. 
Tabla 9. Distribución de frecuencias y porcentajes de Bullying y autoestima en mujeres

\begin{tabular}{|c|c|c|c|c|c|c|c|c|}
\hline \multirow{3}{*}{$\begin{array}{l}\text { Practicas de } \\
\text { Bullying en } \\
\text { Mujeres }\end{array}$} & \multicolumn{6}{|c|}{$\begin{array}{c}\text { Nivel de autoestima en } \\
\text { mujeres }\end{array}$} & \multicolumn{2}{|c|}{ Total } \\
\hline & \multicolumn{2}{|c|}{ Baja } & \multicolumn{2}{|c|}{ Media } & \multicolumn{2}{|c|}{ Alta } & \multirow{2}{*}{$\mathbf{n}$} & \multirow{2}{*}{$\%$} \\
\hline & $n$ & $\%$ & $n$ & $\%$ & $n$ & $\%$ & & \\
\hline Prácticas de Bullying & 30 & 17,3 & 56 & 32,4 & 25 & 14,5 & 111 & 64,2 \\
\hline Ausencia de Bullying & 17 & 9,8 & 33 & 19,1 & 12 & 6,9 & 62 & 35,8 \\
\hline Total & 47 & 27,2 & 89 & 51,3 & 37 & 21,4 & 173 & 100 \\
\hline
\end{tabular}

Se docimó las hipótesis aplicando la Prueba de Friedman (Chi cuadrado de Pearson), obteniendo en varones 10,526 con un p-valor de 0,001 y en la mujeres 52,509 con un $p$-valor de 0,000 ; lo cual indica que existen evidencias estadísticas, para señalar la relación altamente significativa entre el Bullying y la Autoestima en las estudiantes de ambos sexos de la Universidad Nacional José Faustino Sánchez Carrión, presentándose una mayor proporción de práctica de bullying en las estudiantes mujeres.

\section{DISCUSIÓN}

El $57,3 \%$ señala que existen prácticas permanentes de Bullying entre los estudiantes de las Escuelas Profesionales estudiadas, contra un $42,7 \%$ que manifiestan no practicar el Bullying, resultado que nos parece alto, sin embargo una de las conclusiones a que se arribó en la investigación de Benítez, Muñoz, Fernández, y Berbén (2005) sobre Conocimiento y actitud del maltrato entre alumnos (Bullying) de los futuros docentes de educación infantil, primaria y secundaria, en la Universidad de Granada-España, cuyos resultados demandaron la formación de equipos para trabajar con víctimas, agresores, familias y observadores. Del mismo modo, quedó latente la necesidad de trabajar para prevenir el problema y no centrarse solamente en paliar sus efectos. Aunque esta investigación no habla explícitamente de la existencia del bullying, no obstante creemos que dichos sujetos de la muestra si percibían la presencia de este fenómeno.

Ante un $57,3 \%$ de presencia de bullying en la Universidad Nacional José Faustino Sánchez Carrión consideramos pertinente desarrollar proyectos pre y post test en el ámbito universitario para trabajar dimensiones psicológicas como: manejo de control de impulsos, desarrollo de la empatía, tolerancia, mejoramiento, inteligencia emocional, teniendo como antecedente practico el estudio realizado por Romero, Cruz, Gallardo y Peñacoba (2013). Sobre Cómo promocionar la salud y el bienestar en la comunidad universitaria. Universidad Rey Juan Carlos. En la que encontraron evoluciones significativas en cuanto a las variables como autoeficacia, autoestima afecto positivo y negativo señalándose implicaciones prácticas de esta iniciativa como un medio de promocionar la salud y el bienestar en el contexto universitario.

Con respecto a la Autoestima, tenemos que el $53 \%$ tienen nivel de autoestima media que se caracteriza por una personalidad equilibrada en cuanto al manejo de sus pensamientos, impulsos, conductas, emociones, etc. de los cuales el $29,3 \%$ señalan la existencia de práctica de bullying; sobre el tema, Bonet (1997) nos dice que la autoestima es un conjunto de actitudes que dependen de las percepciones, pensamientos, evaluaciones, sentimientos y tendencias de comportamiento dirigidas hacia nosotros mismos, hacia nuestra manera de ser y de comportarnos, y hacia los rasgos de nuestro cuerpo y nuestro carácter; por otro lado el $25,4 \%$ manifiesta autoestima baja de los cuales el $14,2 \%$ refieren la práctica de bullying. Si consideramos en ambos casos existe un porcentaje significativo que señalan practicar la agresión en el ámbito universitario.

Con respecto a la edad de los estudiantes, se encontró que un $93,5 \%$ se encuentra en la etapa de la juventud, es decir entre los 18 y 35 años, de los cuales el $68,7 \%$ se ubican en el rol de agresores y si tenemos que un $57,3 \%$ manifiesta practicar el bullying entonces se estaría concluyendo que un porcentaje significativo de jóvenes practica bullying, lo que nos lleva a preguntarnos a que se debe este fenómeno si se tiene en cuenta que en las características emocionales, de control de impulsos, de manejo de conflictos son más adecuados en esta etapa si la comparamos con la adolescencia, al respecto Álvarez (2013) refiere que los jóvenes en esta etapa integran la lógica con la intuición y la emoción, los hechos con las ideas contradictorias y se encuentran en el máximo rendimiento. Por lo que quedaría pendiente averiguar porque se da esta práctica, puesto que en esta tienen un mejor de razonamiento.

Con respecto a la frecuencia del bullying el $72,8 \%$ manifiestan haber agredido alguna vez a sus compañeros, el $75,4 \%$ señalan haber recibido Bullying de parte de sus compañeros y 
$68,5 \%$ menciona que nunca interviene en un agresión donde él es solo observador, estos porcentajes consideramos bastante altos, pues en todos los casos pasa el $50 \%$, además los estudiantes no perciben estas prácticas como bullying mientras las estudiantes sí perciben la presencia de bullying.

Se concluyó que existe relación entre el Bullying y la Autoestima en los estudiantes de la Universidad Nacional José Faustino Sánchez Carrión.

\section{AGRADECIMIENTO}

Al Decano de la Facultad de Educación quien autorizó la realización de la investigación. A todos aquellos estudiantes de las Escuelas Profesionales que accedieron a la aplicación de los instrumentos de recolección de datos y que permitió arribar a los resultados antes mencionados.

\section{REFERENCIAS BIBLIOGRÁFICAS}

Alvarez, N. (2013). Desarrollo cognoscitivo en la adultez. Recuperado de: https://es.slideshare.net/pei.ac01/expco g-a.

Bandura, A., Ross, D., \& Ross, S. A. (1963). Imitation of film-mediated aggressive models. The Journal of Abnormal and Social Psychology, 66(1), 3.

Bandura, A., \& Mischel, W. (1965). Modifications of self-imposed delay of reward through exposure to live and symbolic models. Journal of personality and social psychology, 2(5), 698.

Bandura, A. (1973). Institutionally sanctioned violence. Journal of Clinical Child Psychology 2(3) 23-24.

Bandura, A. (1973). Aggression: A social learning analysis. Prentice-Hall.

Moreno Martín, F. (1999). Violence in couples. Revista panamericana de salud pública, 5(4-5), 245-258.

Bandura, A. \& Ribes, E. (1975). Modificación de conducta. Análisis de la agresión y la delincuencia. México: Trillas.
Benítez, J., Muñoz, Fernández, M. \& Berbén, A. (2005). Conocimiento y actitud del maltrato entre alumnos (Bullying) de los futuros docentes de educación infantil, primaria y secundaria. Revista de Enseñanza Universitaria, 26,71-86.

Bonet, J. (1997) .Sé amigo de ti mismo: manual de autoestima. Cantabria, España: Sal Terrae.

Cerezo, F. (2001). La violencia en las aulas. Análisis y propuestas de intervención. Madrid, España: Pirámide.

Cerezo, F. (2009). Bullying: análisis de la situación en las aulas españolas. International Journal of Psychology and Psychological Therapy, 9(3), 383-394.

García, J., Moncada, R. \& Quinteros, J. (2013). El Bullying y el suicidio en el escenario universitario. Revista Colombiana de Ciencias Sociales. 4 (2), 298-310. $\mathrm{R}$ e c u p e r a d o d e : http://www.funlam.edu.co/revistas/index .php/RCCS/article/view/1153/1035

Gómez-Talavera, E. (2013). Nivel de agresión entre pares de estudiantes de una Universidad de Lima Metropolitana. Horiz. Med. [online]. oct./dic. 2013, 13(4) [citado 21 Octubre 2014], p.32-37. Disponible en la World Wide Web:

http://revistas.concytec.gob.pe/scielo.p hp? script $=$ sci arttext\&pid $=$ S1727$558 \times 2013000400005 \& \mathrm{lng}=e s \& \mathrm{nrm}=\mathrm{iso}$ $>$. ISSN 1727-558X.

González-Forteza, C., García, G., MedinaMora, E., Sánchez, M. (1998). Indicadores psicosociales predictores de ideación suicida en dos generaciones de estudiantes universitarios. Salud mental, 21(3), 1-9. Instituto Mexicano de Psiquiatría.

Loeber, R., \& Dishion, T. (1983). Early predictors of male delinquency: A review. Psychological bulletin, 94(1), 68.

Moreno Martín, F. (1999). Violence in couples. Revista panamericana de salud pública, 5(4-5), 245-258. 
Olweus, D. (1993). Acoso escolar,"bullying”, en las escuelas: hechos e intervenciones. Centro de investigación para la Promoción de la Salud, Universidad de Bergen, Noruega, 2.

Patterson, G. R., \& Stouthamer-Loeber, M. (1984). The correlation of family management practices and delinquency. Child development, 1299-1307.

Patterson, G. R. (1986). Performance models for antisocial boys. American psychologist, 41(4), 432.

Romero, A., Cruz, S., Gallardo, C. \& Peñacoba, C. (2013). Cómo promocionar la salud y el bienestar en la comunidad universitaria. Universidad Rey Juan Carlos, universidad saludable. Revista Iberoamericana de Psicología y Salud, 4(2), 49-64.
Tarazona, D. (2005). Autoestima, satisfacción con la vida y condiciones de habitabilidad en adolescentes estudiantes de quinto año de media. Un estudio factorial según pobreza y sexo. Revista de Investigación en Psicología, 8(2), 57-65.

Vásquez, A., Jiménez, R. \& Vásquez, R. (2004). Escala de autoestima de Rosemberg: fiabilidad y validez en población clínica española. Apuntes de Psicología Colegio Oficial de Psicología Andalucía Occidental y Universidad de Sevilla, 22(2), 247-255.

\section{Correo electrónico:}

maritmar2012@hotmail.com

Revisión de pares:

Recibido: 08-08-2016

Aceptado: 20-12-2016 